1

2

\title{
Stress, Intertemporal Choice, and Mitigation Behavior During the COVID-19 Pandemic
}

\author{
Mayank Agrawal* \\ Princeton Neuroscience Institute and Department of Psychology, Princeton University \\ *Corresponding Author: mayank.agrawal@ princeton.edu \\ Joshua C. Peterson \\ Department of Computer Science, Princeton University \\ Jonathan D. Cohen \\ Princeton Neuroscience Institute and Department of Psychology, Princeton University \\ Thomas L. Griffiths \\ Departments of Psychology and Computer Science, Princeton University
}




\begin{abstract}
Delayed gratification refers to the willingness to sacrifice smaller, short-term reward for greater, long-term reward. There are suggestions that this proclivity may be impacted by stress and can be predictive of other real-world behaviors. In this article, we outline four large-scale online experiments (total $N=12,906$ ) we conducted over the course of the COVID-19 pandemic, in which we asked individuals to perform Money Earlier or Later (MEL) decisions (e.g. $\$ 5$ today versus $\$ 10$ tomorrow), and to also report stress measures and pandemic mitigation behaviors. We found that behavior in the MEL paradigm correlates with self-reported stress, and that these measures predict the extent of social distancing during the COVID-19 pandemic despite having opposite effects.

Keywords: delayed gratification, intertemporal choice, delay discounting, stress, COVID-19
\end{abstract}




\section{Stress, Intertemporal Choice, and Mitigation Behavior During the COVID-19 Pandemic}

Delayed gratification is an important focus of research, given its potential relationship to forms of behavior, such as savings, susceptibility to addiction, and pro-social behaviors (Mischel, 1974; Funder, Block, \& Block, 1983). While there has been substantial debate about the validity of experimental paradigms that have been used to study deferred gratification (e.g. Mischel, Shoda, \& Rodriguez, 1989), and the interpretation of their results (Benjamin et al., 2020; Cohen, Ericson, Laibson, \& White, 2020), it is clear that humans are often capable of sacrificing immediate rewards for greater long term benefits, and that this proclivity can be predictive of other real-world behaviors. Behavioral scientists refer to this form of decision making as intertemporal choice (ITC) (Samuelson, 1937; Ainslie, 1975; Laibson, 1997; Frederick, Loewenstein, \& O'donoghue, 2002), and have studied it using tasks in which people must decide between receiving a smaller, sooner reward versus a larger, later reward (e.g. $\$ 5$ today versus $\$ 10$ tomorrow). A consistent finding is that an individual's choices in such paradigms can be predictive of behaviors such as alcohol consumption (Vuchinich \& Simpson, 1998), drug use (Kirby, Petry, \& Bickel, 1999), grades (Kirby, Winston, \& Santiesteban, 2005), texting while driving (Hayashi, Russo, \& Wirth, 2015), and smoking (Ohmura, Takahashi, \& Kitamura, 2016).

Stress has been hypothesized as one variable that affects ITC behavior, with greater stress being proposed to lead to more impulsive behavior (Haushofer \& Fehr, 2014). However, the evidence for this has largely been mixed (Lempert, Porcelli, Delgado, \& Tricomi, 2012; Haushofer et al., 2013; Kimura et al., 2013; Delaney, Fink, \& Harmon, 2014; Riis-Vestergaard, van Ast, Cornelisse, Joëls, \& Haushofer, 2018) and has often been restricted to artificial laboratory settings. In the study reported here, we took the opportunity to evaluate whether a natural stressor, the COVID-19 pandemic (Carstensen, Shavit, \& Barnes, 2020), modulated ITC decisions. Furthermore, we hypothesized that mitigation behavior (i.e. the extent of social distancing and mask use) during the pandemic might also reflect a form of intertemporal choice: quicker and more aggressive adoption of mitigation behaviors, though expensive in the short-term, is more likely to lead to better long-term prospects (i.e. faster control of the virus, and thus 
quicker lifting of restrictions). Motivated by these considerations, we conducted four large-scale experiments over the course of the COVID-19 pandemic, in which we collected participants' responses to Money Earlier or Later (MEL) decisions (Frederick et al., 2002; Marzilli Ericson, White, Laibson, \& Cohen, 2015) as well as self-report indicators of stress and mitigation.

\section{Results}

\section{Individual Measures}

Delay discounting (Samuelson, 1937; Ainslie, 1975; Laibson, 1997; Frederick et al., 2002) is the canonical framework to model decision-making in MEL paradigms. Here, the value $v(\cdot)$ of option $o$ with reward $r$ and time delay $t$ is formalized as

$$
v(o)=r \cdot f(t)
$$

in which $f(t)$ represents the discount factor. A hyperbolic function (e.g. Ainslie, 1975) is used to define the discount factor

$$
f(t)=\frac{1}{1+\delta t} .
$$

Larger discount rates (i.e. value of $\delta$ ) are a preference for the smaller, sooner reward and thus indicate greater impulsivity. Once $v\left(o_{1}\right)$ and $v\left(o_{2}\right)$ are computed, the participant selects $o_{1}$ as their choice $c$ with probability

$$
P\left(c=o_{1} \mid v_{o_{1}}, v_{o_{2}}, \tau\right)=\frac{1}{1+e^{-\tau\left(v_{o_{2}}-v_{o_{1}}\right)}} .
$$

\section{Parameters $\delta$ and $\tau$ can be fit with respect to each participant or once across an entire dataset. ${ }^{1}$ For our purposes, we fit discount rates for each individual's set of 200 decisions $^{2}$. Discount rates were then log-transformed for all analyses in order to normalize the variable. We also excluded the}

\footnotetext{
${ }^{1}$ Alternate delay discounting models in the literature include exponential (Samuelson, 1937) and quasi-hyperbolic discounting (Laibson, 1997). Following the recent standard in the intertemporal choice literature (e.g. Hardisty, Fox-Glassman, Krantz, \& Weber, 2011), we focus on the hyperbolic discount rates.

${ }^{2}$ Fitting discount rates from participants' data was done using SciPy (Virtanen et al., 2020).
} 
outer $5 \%$ (i.e. include those between the 2.5 th and 97.5 th percentiles) as a safeguard against any outliers.
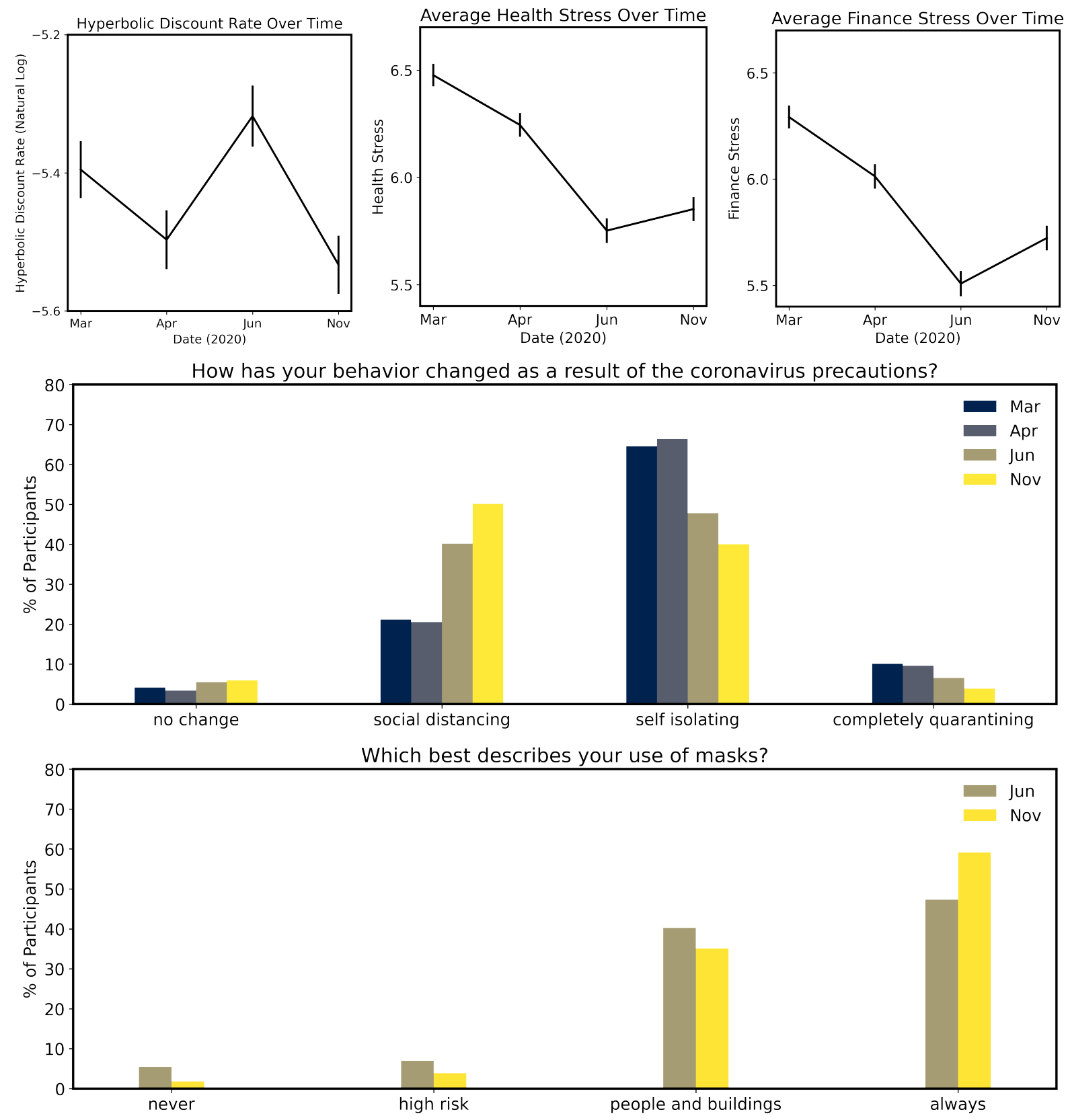

Figure 1. Measures throughout the COVID-19 pandemic. (Top) Average discount rate, health and financial stress measures over time. (Bottom) Distribution of mitigation behaviors over time. four collected datasets. Discount rates oscillated between higher and lower values, whereas the 
stress measures steadily decreased except for a small uptick in Novemeber (immediately prior to the 2020 U.S. election). The middle and bottom panels of Figure 1 report the mitigation behavior of participants. Most participants were self-isolating (i.e., not leaving their home except for purchasing essentials) during the beginning stages, and shifted towards resuming normal activities with social distancing in the later months. Mask use (which was only collected for the last two datasets) was consistently high, with participants increasing use in the last dataset.

\section{Correlations Between Measures}

Next, we evaluated the extent to which discount rates correlated with the self-reported stress measures. The top panel of Figure 2 shows the average (log-transformed) discount rate for each level of stress measure. A hierarchical Bayesian linear regression model ${ }^{3}$ specifying individuals as random effects revealed a significant positive relationship between the discount rates and health stress $(\beta=0.02,95 \% \mathrm{CI}=[0.00,0.04])$, and between discount rates and financial stress $(\beta$ $=0.04,95 \% \mathrm{CI}=[0.03,0.06])$. Thus, we find that higher impulsivity is indeed associated with greater stress, aligning with previous work suggesting that stress can modulate ITC behavior (e.g. Haushofer \& Fehr, 2014). This effect size, seemingly small but significant with a sufficiently large sample, may help account for the disparate findings in the literature relating stress with discount rates (Lempert et al., 2012; Haushofer et al., 2013; Kimura et al., 2013; Delaney et al., 2014; Haushofer \& Fehr, 2014; Riis-Vestergaard et al., 2018).

However, to most accurately interpret the effect size, we plot in Figure 2 the individual discount curves (i.e. Eq. 2) for the mean discount rate at each level of stress. These curves allow us to predict how ITC decision-making is expected to change as a function of stress level. Consider the option of choosing between $\$ 100$ today versus $\$ 110$ in 20 days. Those with the highest health stress are predicted to choose the larger, later option $42 \%$ of the time, whereas the least stressed individuals are predicted to choose it $71 \%$ of the time. Individuals with the highest

\footnotetext{
${ }^{3}$ These analyses were done through the brms package (Bürkner, 2017), which uses a Stan (Carpenter et al., 2017) backend.
} 


\section{Stress vs. Discount Rate}
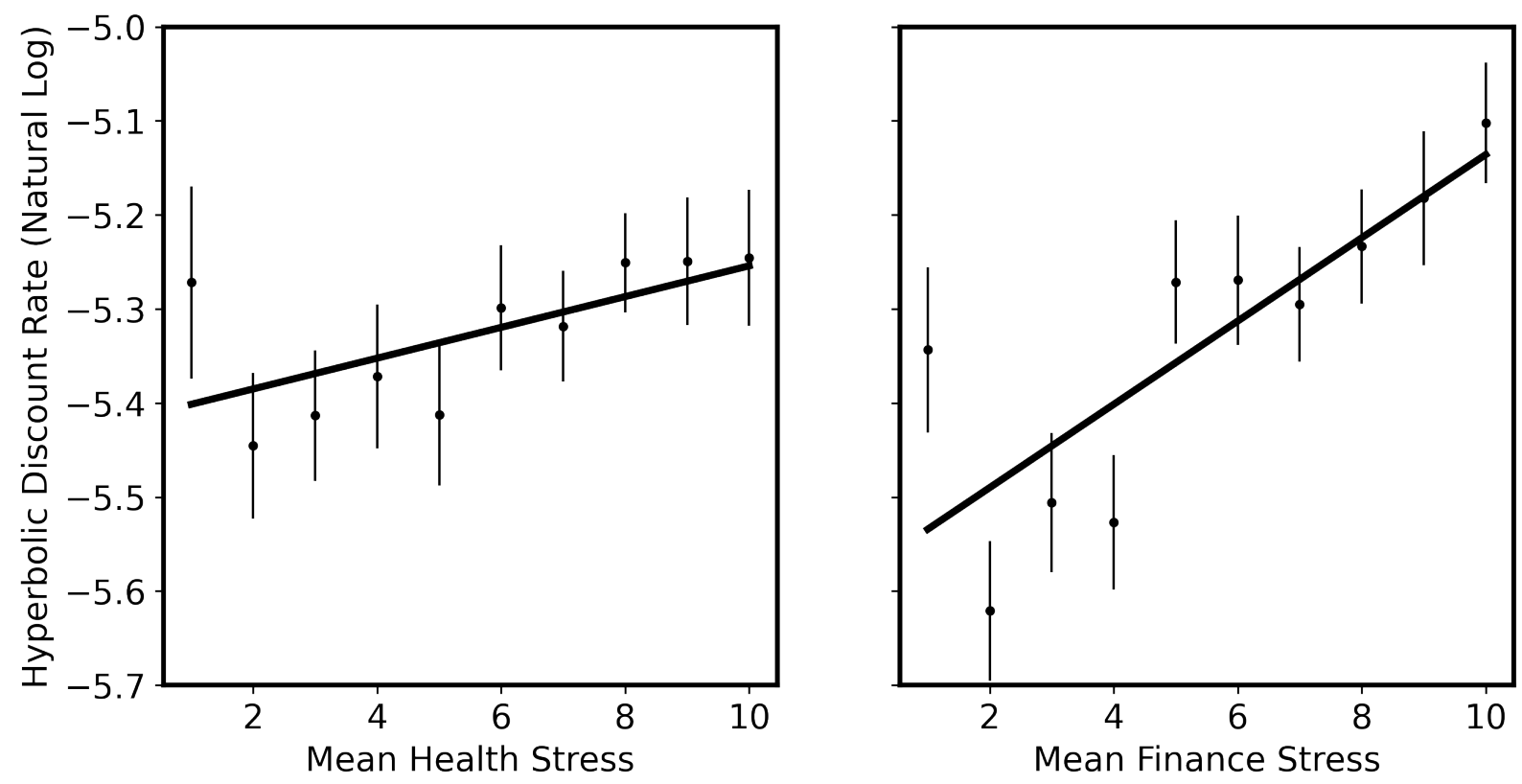

Health Stress

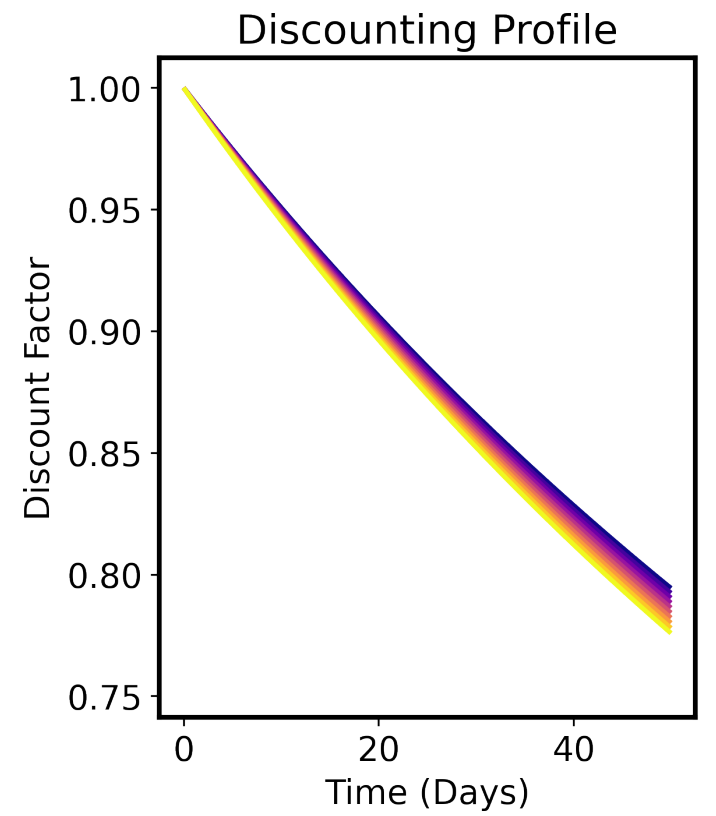

Finance Stress Discounting Profile
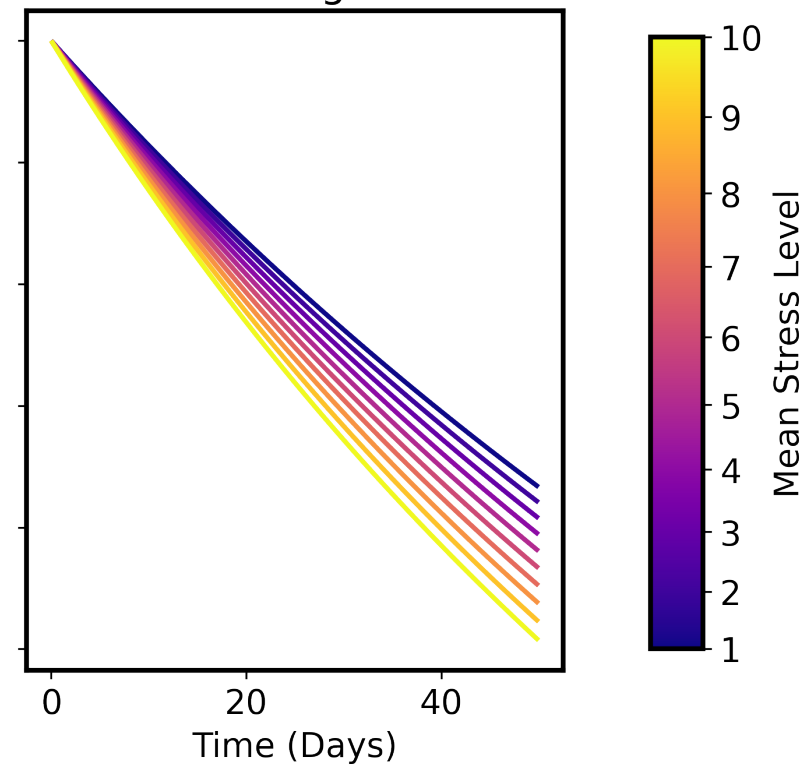

Figure 2. (Top) Average discount rates for each response level of stress. (Bottom) Average hyperbolic discount profiles for each response level of stress. 
which was the median across the dataset).

Finally, we evaluated the extent to which stress measures and discount rates predict mitigation behavior throughout the pandemic. The idea here is that, if social distancing and/or mask use reflects a delayed gratification decision, we should see lower discounting (less impulsive) individuals take more aggressive precautions. Mitigation behaviors were categorical and likely to be unevenly spaced, and thus we modeled responses with a Bayesian ordered logistic regression model (e.g. Liddell \& Kruschke, 2018). First, we linearly mapped the independent variables of interest onto a one-dimensional infinite latent space, and then split this space into intervals that defined the (ordered) categorical responses. We let the intervals be modulated by random effects based on the time of collection in order to allow for changing mitigation norms over time while maintaining the qualitative analysis of interest. Individuals were also modeled as random effects.

For every mitigation behavior, we ran seven unique models, each corresponding to a nontrivial subset of the three coefficients of interest (discount rate, health stress, financial stress). Table 1 reports the fitted coefficients of these models, and suggests that discount rate and health stress jointly predict the extent of social distancing. However, these predictors have opposite effects. Lower discount rate (less impulsivity) predicts more aggressive mitigation behavior, while greater health stress predicts more aggressive mitigation behavior. (This relationship between impulsivity and mitigation behavior makes sense under the interpretation that more aggressive mitigation behaviors are more costly in the short-term, but more valuable in the long-run). These opposite effects of our predictors are especially intriguing since we previously demonstrated in Figure 2 that they are positively correlated. Thus, the downstream effects of stress on social distancing may be more complex than a simple, linear relationship.

Furthermore, we note that while health stress and financial stress independently predict mask use, only the effect of health stress remains statistically significant when put in a joint model. Discount rates did not significantly predict mask use, even when used as the sole predictor. While we cannot be certain why, one conjecture is that mask use is largely affected by 
Table 1

Different Model Fits for Predicting Mitigation Behavior

Social Distancing Mask Use

Variables Coefficient $95 \%$ CI Coefficient $95 \%$ CI

\begin{tabular}{|c|c|c|c|c|}
\hline Discount Rate & -0.04 & {$[-0.07,-0.01]$} & 0.05 & {$[-0.02,0.11]$} \\
\hline Health Stress & 0.30 & {$[0.27,0.34]$} & 0.42 & {$[0.35,0.49]$} \\
\hline Financial Stress & 0.14 & {$[0.11,0.17]$} & 0.18 & {$[0.12,0.24]$} \\
\hline Discount Rate & -0.06 & {$[-0.09,-0.02]$} & 0.02 & {$[-0.04,0.09]$} \\
\hline Health Stress & 0.31 & {$[0.27,0.34]$} & 0.42 & {$[0.35,0.49]$} \\
\hline Discount Rate & -0.05 & {$[-0.09,-0.02]$} & 0.04 & {$[-0.02,0.11]$} \\
\hline Financial Stress & 0.15 & {$[0.12,0.17]$} & 0.18 & {$[0.12,0.25]$} \\
\hline Health Stress & 0.30 & {$[0.26,0.33]$} & 0.46 & {$[0.38,0.55]$} \\
\hline Financial Stress & 0.01 & {$[-0.02,0.04]$} & -0.05 & {$[-0.11,0.01]$} \\
\hline Discount Rate & -0.06 & {$[-0.09,-0.02]$} & 0.03 & {$[-0.03,0.09]$} \\
\hline Health Stress & 0.30 & {$[0.26,0.34]$} & 0.47 & {$[0.39,0.56]$} \\
\hline Financial Stress & 0.01 & {$[-0.02,0.04]$} & -0.06 & {$[-0.12,0.01]$} \\
\hline
\end{tabular}

politics (e.g. Xu \& Cheng, 2021).

The Supplement reports similar analyses as Table 1, but for each individual dataset. In the individual datasets, effect sizes remained similar, but confidence intervals were wider, presumably 
due to the decreased sample size.

\section{Discussion}

The relationship between stress and intertemporal choice (ITC) has been a longstanding question in the literature on human decision-making. We leveraged a natural stressor (the COVID-19 pandemic) and large online samples to discover there exists a small but significant positive relationship between log-transformed discount rates and perceptions of health and financial stress. We used example MEL dilemmas to illustrate the magnitude of this effect, demonstrating how each increase in stress level affects the aggregate response. Extreme stress can have a meaningful effect on the extent to which people discount future rewards when making financial decisions.

Exploring this question during a pandemic also provided an opportunity to contribute to the existing body of work suggesting that ITC behavior can be predictive of real-world behaviors (Kirby et al., 1999; Vuchinich \& Simpson, 1998; Kirby et al., 2005; Hayashi et al., 2015; Ohmura et al., 2016). We found that degree of social distancing, but not mask use, was statistically significantly correlated with discount rates in financial decision-making. This result may be of interest to public health officials as they try to plan responses for similar situations in the future. In particular, the stresses associated with a pandemic might be expected to attenuate future-oriented thinking, leading people to make decisions that favor short-term payoffs. Planning for such shifts in decision-making will result in more effective modeling and interventions.

We hope these results further motivate interest into discount rates as an important psychological variable to study. It was not surprising that financial stress had a stronger correlation with discount rates than did health stress. But, the fact that discount rate maintained a statistically significant influence when put in a joint model predicting social distancing behavior suggests that the metric encodes unique information that is not captured in the stress measures. These results are consistent with the idea that there may be a psychological factor underlying ITC decision-making that has a broader impact on behavior (e.g. Viscusi \& Moore, 1989; Coile, 
Diamond, Gruber, \& Jousten, 2002; Becker, Deckers, Dohmen, Falk, \& Kosse, 2012; Golsteyn, Grönqvist, \& Lindahl, 2014; Benjamin et al., 2020).

\section{Methods}

\section{Participants}

Four large-scale experiments were run via Amazon's Mechanical Turk with recruitment of participants in the United States via CloudResearch (Litman, Robinson, \& Abberbock, 2017) on March 26, $2020(N=3,335)$; April 15, $2020(N=3,195)$; June 30, $2020(N=3,216)$; and November 2, $2020(N=3,160)$. Each of these samples included participants from all 50 states and the District of Columbia, with ages varying from 18 to 89 (see Supplement for detailed demographic analyses). The experiments were conducted until every unique MEL problem specification (defined below) received twenty responses. Participants were required to have received at least a $95 \%$ approval rate on a minimum of 100 tasks. Once a participant completed one experiment, they were e-mailed at the start of subsequent experiments to encourage repeated measures at different time points throughout the pandemic. The Supplement outlines two control experiments validating the experimental paradigm and the use of this crowdsourcing service. We also report there the participant overlap between datasets.

This study was approved by the Institutional Review Board at Princeton University. All participants provided informed consent and were compensated $\$ 2.50$ for each experiment.

\section{Materials and Procedure}

Participants made choices in 200 MEL scenarios. Following the format of Marzilli Ericson et al. (2015), participants were randomly placed into one of the five framing conditions defined below:

\section{Absolute Difference, Delay Framing:} $\$ 5$ today vs. $\$ 5$ plus an additional $\$ 5$ in 4 weeks 


\section{Relative Difference, Delay Framing:}

$\$ 5$ today vs. $\$ 5$ plus an additional $100 \%$ in 4 weeks

\section{Standard Money Earlier or Later (MEL) Format:}

$\$ 5$ today vs. $\$ 10$ in 4 weeks

\section{Absolute Difference, Speedup Framing:}

$\$ 10$ in 4 weeks vs. $\$ 10$ minus $\$ 5$ today

\section{Relative Difference, Speedup Framing:}

$\$ 10$ in 4 weeks vs. $\$ 10$ minus $50 \%$ today

195 unique decisions were then sampled from the problem parameters:

\section{Absolute Value Difference:}

$\$ 0.10, \$ 0.50, \$ 1.00, \$ 2.00, \$ 5.00, \$ 10.00$,

$\$ 25.00, \$ 50.00, \$ 100.00, \$ 500.00, \$ 1000.00$

\section{Relative Value Difference:}

$1 \%, 5 \%, 10 \%, 25 \%, 50 \%, 100 \%, 200 \%, 300 \%$

\section{Start Time:}

today, 1 day, 2 days, 4 days, 7 days, 14 days, 21 days,

50 days

\section{Time Difference:}

1 day, 2 days, 4 days, 7 days, 14 days, 21 days,

50 days

For example, if the absolute value difference was $\$ 5.00$, the relative value difference $100 \%$, the start time 2 days, the time difference 7 days, and the framing condition standard MEL format, then the participant was asked to choose between $\$ 5$ in 2 days and $\$ 10$ in 9 days. Responses across the five framing conditions were collapsed prior to analyses.

An additional 5 dilemmas were included in which the sooner reward was larger and the later reward was smaller. These served as attention checks, in which failure was defined as 
choosing the smaller, later option, and were added to the original 195 dilemmas for a total of 200 dilemmas. For any given dataset, participants were excluded from subsequent analyses if they (1) failed any of the five attention checks; (2) always chose the larger, later (LL) option; or (3) always chose the smaller, sooner (SS) option.

Then, to collect stress measures among our population, we asked participants variants of the principal question of the annual Stress in America survey conducted by the American Psychological Association (American Psychological Association, 2007):

On a scale of 1 to 10 where 1 means you have "little or no stress" and 10 means you have "a great deal of stress," how would you rate your current level of stress about

1. the health of yourself and your friends/family?

2. the finances of yourself and your friends/family?

3. the upcoming U.S. presidential election?

The election question was only asked in the November dataset.

Second, to assess mitigation behavior, we asked:

Which best describes how your behavior has changed as a result of the coronavirus precautions? (single choice)

- No change

- Social distancing (e.g., maintaining 6 feet of distance), but otherwise no change

- Self-quarantined except for buying essentials (food, supplies, etc.)

- Completely self-quarantined (relying on delivery)

We collected mask use data in the last two datasets:

Which best describes your use of masks? (single choice)

- Always, when outside home 
- When close to people or inside buildings

- Only inside high-risk environments (such as hospitals)

- Never

Last, to gauge predictions about increases in infections, we asked:

The current number of confirmed coronavirus (COVID-19) infections in the US is approximately $X, X X X$ as of today. What would you estimate this number to be:

Tomorrow

One week from now

One month from now

The estimated number of confirmed coronavirus infections in the U.S. was provided by the Johns Hopkins Coronavirus Resource Center ${ }^{4}$. These prediction data have not been analyzed.

\section{Acknowledgements}

This work is supported by NSF RAPID AWD1006491 and grants from the NOMIS

Foundation and the Princeton Data-Driven Social Science Initiative. M.A. is supported by the National Defense Science and Engineering Graduate Fellowship Program.

${ }^{4}$ https://coronavirus.jhu.edu/ 
References

Ainslie, G. (1975). Specious reward: a behavioral theory of impulsiveness and impulse control. Psychological Bulletin, 82(4), 463.

American Psychological Association. (2007). Stress in america. Retrieved from www.apa.org/pubs/info/reports/2007-stress.doc, 24, 2007.

Becker, A., Deckers, T., Dohmen, T., Falk, A., \& Kosse, F. (2012). The relationship between economic preferences and psychological personality measures. Annu. Rev. Econ., 4(1), $453-478$.

Benjamin, D. J., Laibson, D., Mischel, W., Peake, P. K., Shoda, Y., Wellsjo, A. S., \& Wilson, N. L. (2020). Predicting mid-life capital formation with pre-school delay of gratification and life-course measures of self-regulation. Journal of Economic Behavior \& organization, $179,743-756$.

Bürkner, P.-C. (2017). brms: An r package for bayesian multilevel models using stan. Journal of Statistical Software, 80(1), 1-28.

Carpenter, B., Gelman, A., Hoffman, M. D., Lee, D., Goodrich, B., Betancourt, M., ... Riddell, A. (2017). Stan: A probabilistic programming language. Journal of Statistical Software, $76(1), 1-32$.

Carstensen, L. L., Shavit, Y. Z., \& Barnes, J. T. (2020). Age advantages in emotional experience persist even under threat from the covid-19 pandemic. Psychological Science, 31(11), $1374-1385$.

Chandler, J., Rosenzweig, C., Moss, A. J., Robinson, J., \& Litman, L. (2019). Online panels in social science research: Expanding sampling methods beyond mechanical turk. Behavior Research Methods, 51(5), 2022-2038.

Cohen, J., Ericson, K. M., Laibson, D., \& White, J. M. (2020). Measuring time preferences. Journal of Economic Literature, 58(2), 299-347.

Coile, C., Diamond, P., Gruber, J., \& Jousten, A. (2002). Delays in claiming social security benefits. Journal of Public Economics, 84(3), 357-385. 
Delaney, L., Fink, G., \& Harmon, C. P. (2014). Effects of stress on economic decision-making: Evidence from laboratory experiments.

Frederick, S., Loewenstein, G., \& O'donoghue, T. (2002). Time discounting and time preference: A critical review. Journal of Economic Literature, 40(2), 351-401.

Funder, D. C., Block, J. H., \& Block, J. (1983). Delay of gratification: some longitudinal personality correlates. Journal of Personality and Social Psychology, 44(6), 1198.

Golsteyn, B. H., Grönqvist, H., \& Lindahl, L. (2014). Adolescent time preferences predict lifetime outcomes. The Economic Journal, 124(580), F739-F761.

Hardisty, D. J., Fox-Glassman, K., Krantz, D., \& Weber, E. U. (2011). How to measure discount rates? an experimental comparison of three methods. An Experimental Comparison of Three Methods.

Haushofer, J., Cornelisse, S., Seinstra, M., Fehr, E., Joëls, M., \& Kalenscher, T. (2013). No effects of psychosocial stress on intertemporal choice. PloS One, 8(11), e78597.

Haushofer, J., \& Fehr, E. (2014). On the psychology of poverty. Science, 344(6186), 862-867.

Hayashi, Y., Russo, C. T., \& Wirth, O. (2015). Texting while driving as impulsive choice: A behavioral economic analysis. Accident Analysis \& Prevention, 83, 182-189.

Kimura, K., Izawa, S., Sugaya, N., Ogawa, N., Yamada, K. C., Shirotsuki, K., .. Hasegawa, T. (2013). The biological effects of acute psychosocial stress on delay discounting. Psychoneuroendocrinology, 38(10), 2300-2308.

Kirby, K. N., Petry, N. M., \& Bickel, W. K. (1999). Heroin addicts have higher discount rates for delayed rewards than non-drug-using controls. Journal of Experimental Psychology: General, 128(1), 78.

Kirby, K. N., Winston, G. C., \& Santiesteban, M. (2005). Impatience and grades: Delay-discount rates correlate negatively with college gpa. Learning and Individual Differences, 15(3), $213-222$.

Laibson, D. (1997). Golden eggs and hyperbolic discounting. The Quarterly Journal of Economics, 112(2), 443-478. 
Lempert, K. M., Porcelli, A. J., Delgado, M. R., \& Tricomi, E. (2012). Individual differences in delay discounting under acute stress: the role of trait perceived stress. Frontiers in Psychology, 3, 251.

Liddell, T. M., \& Kruschke, J. K. (2018). Analyzing ordinal data with metric models: What could possibly go wrong? Journal of Experimental Social Psychology, 79, 328-348.

Litman, L., Robinson, J., \& Abberbock, T. (2017). Turkprime. com: A versatile crowdsourcing data acquisition platform for the behavioral sciences. Behavior Research Methods, 49(2), 433-442.

Marzilli Ericson, K. M., White, J. M., Laibson, D., \& Cohen, J. D. (2015). Money earlier or later? simple heuristics explain intertemporal choices better than delay discounting does. Psychological Science, 26(6), 826-833.

Mischel, W. (1974). Processes in delay of gratification. In Advances in experimental social psychology (Vol. 7, pp. 249-292). Elsevier.

Mischel, W., Shoda, Y., \& Rodriguez, M. I. (1989). Delay of gratification in children. Science, 244(4907), 933-938.

Ohmura, Y., Takahashi, T., \& Kitamura, N. (2016). Discounting delayed and probabilistic monetary gains and losses by smokers of cigarettes. In Behavioral economics of preferences, choices, and happiness (pp. 179-196). Springer.

Palan, S., \& Schitter, C. (2018). Prolific. ac-a subject pool for online experiments. Journal of Behavioral and Experimental Finance, 17, 22-27.

Riis-Vestergaard, M. I., van Ast, V., Cornelisse, S., Joëls, M., \& Haushofer, J. (2018). The effect of hydrocortisone administration on intertemporal choice. Psychoneuroendocrinology, 88 , $173-182$.

Samuelson, P. A. (1937). A note on measurement of utility. The Review of Economic Studies, $4(2), 155-161$.

Virtanen, P., Gommers, R., Oliphant, T. E., Haberland, M., Reddy, T., Cournapeau, D., ... others (2020). Scipy 1.0: fundamental algorithms for scientific computing in python. Nature 
Methods, 17(3), 261-272.

Viscusi, W. K., \& Moore, M. J. (1989). Rates of time preference and valuations of the duration of life. Journal of public economics, 38(3), 297-317.

Vuchinich, R. E., \& Simpson, C. A. (1998). Hyperbolic temporal discounting in social drinkers and problem drinkers. Experimental and Clinical Psychopharmacology, 6(3), 292.

$\mathrm{Xu}, \mathrm{P} .$, \& Cheng, J. (2021). Individual differences in social distancing and mask-wearing in the pandemic of covid-19: The role of need for cognition, self-control and risk attitude. Personality and individual differences, 175, 110706. 


\section{Supplement}

\section{Control Experiments}

To allay recent concerns about the data quality on Amazon's Mechanical Turk, we conducted a direct replication of a previous MEL study (Marzilli Ericson et al., 2015) on three platforms: Amazon's Mechanical Turk, Prolific (Palan \& Schitter, 2018), and a nationally representative sample from CloudResearch's Prime Panels (Chandler, Rosenzweig, Moss, Robinson, \& Litman, 2019). 25 decisions each from 1,000 participants were collected and analyzed. While there were some statistical variation in the fitted (log transformed) hyperbolic discount rates (Mechanical Turk mean: -3.98, SEM: 0.11; Prolific mean: -4.25, SEM: 0.11; Prime Panel mean: -3.46, SEM: 0.12), the qualitative patterns in the three datasets remained the same. A histogram of the distribution is plotted below:

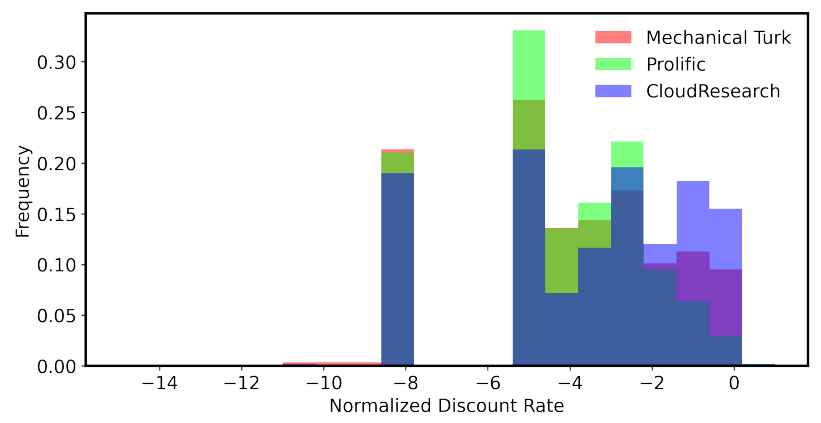

Figure 3. Distribution of hyperbolic discount rates across three crowdsourcing platforms.

Second, to allay concerns about the lack of incentives in our experiments, we conducted a study examining the role of incentives in ITC behavior. Ten fixed questions were chosen:

1. $\$ 0.03$ in 0 days vs. $\$ 0.13$ in 2 days

2. $\$ 0.10$ in 1 day vs. $\$ 0.20$ in 8 days

3. $\$ 0.20$ in 1 day vs. $\$ 0.30$ in 8 days

4. $\$ 0.33$ in 0 days vs. $\$ 1.33$ in 1 day 
Table 2

Sex
5. $\$ 0.40$ in 0 days vs. $\$ 0.50$ in 4 days

6. $\$ 1.00$ in 7 days vs. $\$ 1.50$ in 8 days

7. $\$ 1.00$ in 1 day vs. $\$ 3.00$ in 3 days

8. $\$ 2.00$ in 0 days vs. $\$ 2.10$ in 7 days

9. $\$ 2.00$ in 2 days vs. $\$ 2.50$ in 4 days

10. $\$ 5.00$ in 0 days vs. $\$ 5.50$ in 7 days

200 participants were recruited for the experiment. Half were put in the no incentive condition, and half were put in the incentive condition. Those in the incentive condition were told that one of their choices would be randomly chosen and the money would be bonused to them after the time delay. All ten Chi-squared analyses between the decisions in the no incentive vs. incentive condition were not statistically significant at the $\alpha=0.05$ level.

\section{Demographics}

In Tables 2 to 5, we report the demographic data of the participants after exclusion criteria were applied.

\begin{tabular}{ccccc} 
& Mar & Apr & Jun & Nov \\
\hline Male & $52.0 \%$ & $51.7 \%$ & $50.8 \%$ & $50.3 \%$ \\
Female & $47.8 \%$ & $48.0 \%$ & $48.9 \%$ & $49.4 \%$ \\
Other & $0.2 \%$ & $0.3 \%$ & $0.3 \%$ & $0.3 \%$ \\
\hline
\end{tabular}


Table 3

Education

\begin{tabular}{ccccc} 
& Mar & Apr & Jun & Nov \\
\hline Graduate & $15.7 \%$ & $16.3 \%$ & $16.6 \%$ & $16.1 \%$ \\
Completed College & $46.0 \%$ & $46.9 \%$ & $46.3 \%$ & $46.6 \%$ \\
Some College & $26.8 \%$ & $27.2 \%$ & $26.8 \%$ & $26.3 \%$ \\
Secondary & $11.2 \%$ & $9.4 \%$ & $10.0 \%$ & $10.6 \%$ \\
Primary & $0.4 \%$ & $0.1 \%$ & $0.2 \%$ & $0.2 \%$ \\
None & $0.0 \%$ & $0.0 \%$ & $0.1 \%$ & $0.0 \%$ \\
\hline
\end{tabular}

Table 4

Age

\begin{tabular}{ccccc} 
& Mar & Apr & Jun & Nov \\
\hline 18 to 20 & $6.0 \%$ & $7.0 \%$ & $8.1 \%$ & $6.3 \%$ \\
21 to 44 & $66.6 \%$ & $65.0 \%$ & $62.9 \%$ & $62.8 \%$ \\
45 to 64 & $28.1 \%$ & $28.9 \%$ & $30.2 \%$ & $30.4 \%$ \\
65 and over & $4.7 \%$ & $5.2 \%$ & $6.0 \%$ & $6.1 \%$ \\
\hline
\end{tabular}

Table 5

State

\begin{tabular}{cccccc} 
State & Mar & Apr & Jun & Nov & U.S. Census \\
\hline AK & $0.1 \%$ & $0.0 \%$ & $0.1 \%$ & $0.1 \%$ & $0.2 \%$ \\
AL & $1.7 \%$ & $1.7 \%$ & $1.1 \%$ & $1.3 \%$ & $1.5 \%$ \\
AR & $0.6 \%$ & $0.7 \%$ & $0.7 \%$ & $0.7 \%$ & $0.9 \%$ \\
AZ & $2.1 \%$ & $1.9 \%$ & $2.1 \%$ & $1.9 \%$ & $2.2 \%$ \\
CA & $10.4 \%$ & $10.5 \%$ & $9.5 \%$ & $9.4 \%$ & $12.0 \%$
\end{tabular}




\begin{tabular}{|c|c|c|c|c|c|}
\hline $\mathrm{CO}$ & $1.9 \%$ & $1.7 \%$ & $1.7 \%$ & $2.0 \%$ & $1.8 \%$ \\
\hline CT & $1.3 \%$ & $1.3 \%$ & $1.3 \%$ & $1.6 \%$ & $1.1 \%$ \\
\hline DC & $0.2 \%$ & $0.3 \%$ & $0.2 \%$ & $0.1 \%$ & $0.2 \%$ \\
\hline $\mathrm{DE}$ & $0.2 \%$ & $0.3 \%$ & $0.3 \%$ & $0.2 \%$ & $0.3 \%$ \\
\hline FL & $7.6 \%$ & $7.7 \%$ & $7.9 \%$ & $7.8 \%$ & $6.5 \%$ \\
\hline GA & $2.8 \%$ & $3.1 \%$ & $3.1 \%$ & $4.0 \%$ & $3.2 \%$ \\
\hline HI & $0.5 \%$ & $0.8 \%$ & $0.7 \%$ & $0.4 \%$ & $0.4 \%$ \\
\hline IA & $1.2 \%$ & $1.0 \%$ & $1.0 \%$ & $0.8 \%$ & $1.0 \%$ \\
\hline ID & $0.4 \%$ & $0.3 \%$ & $0.5 \%$ & $0.5 \%$ & $0.5 \%$ \\
\hline IL & $4.3 \%$ & $4.2 \%$ & $3.8 \%$ & $3.8 \%$ & $3.9 \%$ \\
\hline IN & $1.9 \%$ & $1.6 \%$ & $1.8 \%$ & $1.6 \%$ & $2.0 \%$ \\
\hline KS & $0.8 \%$ & $0.4 \%$ & $0.8 \%$ & $0.9 \%$ & $0.9 \%$ \\
\hline KY & $1.8 \%$ & $1.4 \%$ & $1.5 \%$ & $2.0 \%$ & $1.4 \%$ \\
\hline LA & $0.9 \%$ & $0.9 \%$ & $1.0 \%$ & $0.9 \%$ & $1.4 \%$ \\
\hline MA & $2.0 \%$ & $1.9 \%$ & $2.1 \%$ & $2.8 \%$ & $2.1 \%$ \\
\hline MD & $1.8 \%$ & $2.0 \%$ & $1.8 \%$ & $2.1 \%$ & $1.8 \%$ \\
\hline $\mathrm{ME}$ & $0.5 \%$ & $0.7 \%$ & $0.4 \%$ & $0.5 \%$ & $0.4 \%$ \\
\hline MI & $3.5 \%$ & $3.3 \%$ & $2.9 \%$ & $2.9 \%$ & $3.0 \%$ \\
\hline $\mathrm{MN}$ & $1.3 \%$ & $1.7 \%$ & $1.7 \%$ & $1.5 \%$ & $1.7 \%$ \\
\hline MO & $1.8 \%$ & $2.6 \%$ & $1.7 \%$ & $2.0 \%$ & $1.9 \%$ \\
\hline MS & $0.7 \%$ & $0.6 \%$ & $0.5 \%$ & $1.0 \%$ & $0.9 \%$ \\
\hline MT & $0.4 \%$ & $0.3 \%$ & $0.3 \%$ & $0.2 \%$ & $0.3 \%$ \\
\hline $\mathrm{NC}$ & $3.5 \%$ & $4.4 \%$ & $4.6 \%$ & $3.8 \%$ & $3.2 \%$ \\
\hline ND & $0.0 \%$ & $0.1 \%$ & $0.0 \%$ & $0.1 \%$ & $0.2 \%$ \\
\hline $\mathrm{NE}$ & $0.6 \%$ & $0.7 \%$ & $0.4 \%$ & $0.8 \%$ & $0.6 \%$ \\
\hline $\mathrm{NH}$ & $0.6 \%$ & $0.6 \%$ & $0.3 \%$ & $0.4 \%$ & $0.4 \%$ \\
\hline NJ & $3.0 \%$ & $3.0 \%$ & $2.9 \%$ & $2.5 \%$ & $2.7 \%$ \\
\hline
\end{tabular}




\begin{tabular}{llllll} 
NM & $0.7 \%$ & $0.5 \%$ & $0.8 \%$ & $0.4 \%$ & $0.6 \%$ \\
NV & $1.3 \%$ & $0.9 \%$ & $1.5 \%$ & $0.9 \%$ & $0.9 \%$ \\
NY & $6.1 \%$ & $5.7 \%$ & $6.3 \%$ & $6.0 \%$ & $5.9 \%$ \\
OH & $4.2 \%$ & $3.7 \%$ & $4.2 \%$ & $4.8 \%$ & $3.6 \%$ \\
OK & $0.8 \%$ & $1.4 \%$ & $0.8 \%$ & $0.6 \%$ & $1.2 \%$ \\
OR & $1.6 \%$ & $1.5 \%$ & $1.6 \%$ & $1.7 \%$ & $1.3 \%$ \\
PA & $5.2 \%$ & $5.7 \%$ & $5.7 \%$ & $5.5 \%$ & $3.9 \%$ \\
RI & $0.3 \%$ & $0.4 \%$ & $0.5 \%$ & $0.3 \%$ & $0.3 \%$ \\
SC & $1.5 \%$ & $1.3 \%$ & $1.3 \%$ & $1.1 \%$ & $1.6 \%$ \\
SD & $0.1 \%$ & $0.3 \%$ & $0.3 \%$ & $0.4 \%$ & $0.3 \%$ \\
TN & $2.1 \%$ & $1.3 \%$ & $1.8 \%$ & $2.4 \%$ & $2.1 \%$ \\
TX & $6.9 \%$ & $5.9 \%$ & $6.8 \%$ & $6.0 \%$ & $8.8 \%$ \\
UT & $0.8 \%$ & $0.8 \%$ & $0.7 \%$ & $0.7 \%$ & $1.0 \%$ \\
VA & $2.2 \%$ & $2.7 \%$ & $3.0 \%$ & $2.9 \%$ & $2.6 \%$ \\
VT & $0.2 \%$ & $0.1 \%$ & $0.1 \%$ & $0.2 \%$ & $0.2 \%$ \\
WA & $2.6 \%$ & $2.3 \%$ & $3.0 \%$ & $2.8 \%$ & $2.3 \%$ \\
WI & $2.3 \%$ & $2.3 \%$ & $2.3 \%$ & $2.1 \%$ & $1.8 \%$ \\
WV & $0.6 \%$ & $0.7 \%$ & $0.6 \%$ & $0.4 \%$ & $0.6 \%$ \\
WY & $0.0 \%$ & $0.1 \%$ & $0.3 \%$ & $0.1 \%$ & $0.2 \%$ \\
\hline
\end{tabular}

\section{Potential Selection Effects}

Here, we address a concern that the Mechanical Turk population potentially changed throughout the course of our experiment due to secondary effects from the pandemic. First, Table 6 reports the overlap in participants between each pairwise dataset. All $N$ are reported after exclusion criteria were applied.

Next, Tables 7 to 10 outlines the main text analyses, but for each individual dataset (as opposed to combining the datasets and using time of collection as a random variable). 
Table 6

Participant Overlap Between Datasets

\begin{tabular}{ccccc} 
Month & N & Month & N & Overlap \\
\hline March & 2174 & April & 2014 & 1017 \\
March & 2174 & June & 1971 & 818 \\
March & 2174 & November & 2050 & 713 \\
April & 2014 & June & 1971 & 821 \\
April & 2014 & November & 2050 & 696 \\
June & 1971 & November & 2050 & 783 \\
\hline
\end{tabular}
model convergence. 
Table 7

March

\begin{tabular}{|c|c|c|c|}
\hline & Social D & tancing & Mask Use \\
\hline Variables & Coefficient & $\mathbf{9 5} \% \mathrm{CI}$ & Coefficient 95\% CI \\
\hline
\end{tabular}

Discount Rate $\quad-0.03 \quad[-0.07,0.02] \quad$ N/A $\quad$ N/A

Health Stress $\quad 0.20 \quad[0.16,0.23] \quad$ N/A $\quad$ N/A

Financial Stress $\quad 0.07 \quad[0.04,0.11] \quad$ N/A $\quad$ N/A

Discount Rate $\quad-0.04 \quad[-0.08,0.01] \quad$ N/A $\quad$ N/A

Health Stress $\quad 0.20 \quad[0.16,0.23] \quad$ N/A N/A

Discount Rate $\quad-0.03 \quad[-0.08,0.01] \quad$ N/A $\quad$ N/A

Financial Stress $\quad 0.08 \quad[0.04,0.11] \quad$ N/A $\quad$ N/A

Health Stress $\quad 0.21 \quad[0.17,0.25] \quad$ N/A $\quad$ N/A

Financial Stress $\quad-0.02 \quad[-0.06,0.02] \quad$ N/A $\quad$ N/A

Discount Rate $\quad-0.03 \quad[-0.08,0.01] \quad$ N/A $\quad$ N/A

Health Stress $\quad 0.21 \quad[0.17,0.25] \quad$ N/A $\quad$ N/A

Financial Stress $\quad-0.02 \quad[-0.05,0.02] \quad$ N/A $\quad$ N/A 
Table 8

April

\begin{tabular}{|c|c|c|c|c|}
\hline \multirow[b]{2}{*}{ Variables } & \multicolumn{2}{|c|}{ Social Distancing } & \multicolumn{2}{|c|}{ Mask Use } \\
\hline & Coefficient & $95 \% \mathrm{CI}$ & Coefficic & $5 \% \mathrm{CI}$ \\
\hline Discount Rate & -0.06 & {$[-0.11,-0.02]$} & N/A & N/A \\
\hline Health Stress & 0.17 & {$[0.13,0.21]$} & N/A & N/A \\
\hline Financial Stress & 0.11 & {$[0.07,0.14]$} & N/A & N/A \\
\hline Discount Rate & -0.07 & {$[-0.11,-0.02]$} & N/A & N/A \\
\hline Health Stress & 0.17 & {$[0.13,0.20]$} & N/A & N/A \\
\hline Discount Rate & -0.07 & {$[-0.12,-0.02]$} & N/A & N/A \\
\hline Financial Stress & 0.11 & {$[0.08,0.14]$} & N/A & N/A \\
\hline Health Stress & 0.15 & {$[0.11,0.19]$} & N/A & N/A \\
\hline Financial Stress & 0.03 & {$[-0.01,0.07]$} & N/A & N/A \\
\hline Discount Rate & -0.07 & {$[-0.11,-0.02]$} & N/A & N/A \\
\hline Health Stress & 0.15 & {$[0.11,0.19]$} & N/A & N/A \\
\hline Financial Stress & 0.04 & {$[-0.00,0.08]$} & N/A & N/A \\
\hline
\end{tabular}


Table 9

June

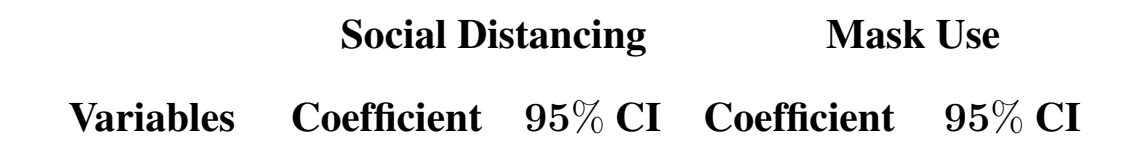

Discount Rate $\quad-0.02 \quad[-0.09,0.04] \quad 0.03 \quad[-0.01,0.07]$

Health Stress $\quad 0.24 \quad[0.21,0.28] \quad 0.25 \quad[0.22,0.28]$

Financial Stress $\quad 0.11 \quad[0.08,0.14] \quad 0.08 \quad[0.05,0.12]$

Discount Rate $\quad-0.03 \quad[-0.07,0.01] \quad 0.02 \quad[-0.03,0.06]$

Health Stress $\quad 0.24 \quad[0.21,0.28] \quad 0.25 \quad[0.22,0.28]$

Discount Rate $\quad-0.03 \quad[-0.07,0.01] \quad 0.02 \quad[-0.02,0.06]$

Financial Stress $\quad 0.11 \quad[0.08,0.14] \quad 0.08 \quad[0.05,0.12]$

Health Stress $\quad 0.25 \quad[0.21,0.29] \quad 0.28 \quad[0.24,0.32]$

Financial Stress $\quad-0.01 \quad[-0.05,0.03] \quad-0.06 \quad[-0.10,-0.02]$

Discount Rate $\quad-0.03 \quad[-0.07,0.01] \quad 0.02 \quad[-0.02,0.06]$

Health Stress $\quad 0.25 \quad[0.21,0.29] \quad 0.28 \quad[0.24,0.33]$

Financial Stress $\quad-0.01 \quad[-0.05,0.03] \quad-0.06 \quad[-0.10,-0.02]$ 
Table 10

November

\begin{tabular}{lcccc} 
& \multicolumn{2}{c}{ Social Distancing } & \multicolumn{2}{c}{ Mask Use } \\
Variables & Coefficient & $\mathbf{9 5 \%}$ CI & Coefficient & $\mathbf{9 5 \%}$ CI
\end{tabular}

Discount Rate $\quad-0.08 \quad[-0.25,0.04] \quad 0.03 \quad[-0.01,0.07]$

$\begin{array}{lllll}\text { Health Stress } & 0.28 \quad[0.24,0.31] & 0.25 & {[0.21,0.28]}\end{array}$

Financial Stress $\quad 0.13 \quad[0.10,0.16] \quad 0.09 \quad[0.06,0.12]$

Discount Rate $\quad-0.05 \quad[-0.09,-0.00] \quad 0.02 \quad[-0.02,0.07]$

$\begin{array}{llll}\text { Health Stress } & 0.28 \quad[0.24,0.31] & 0.25 & {[0.21,0.28]}\end{array}$

Discount Rate $\quad-0.05 \quad[-0.09,-0.00] \quad 0.02 \quad[-0.02,0.07]$

Financial Stress $\quad 0.14 \quad[0.10,0.17] \quad 0.09 \quad[0.06,0.12]$

Health Stress $\quad 0.27 \quad[0.23,0.31] \quad 0.26 \quad[0.22,0.30]$

Financial Stress $\quad 0.01 \quad[-0.02,0.05] \quad-0.03 \quad[-0.06,0.01]$

Discount Rate $\quad-0.05 \quad[-0.10,-0.01] \quad 0.02 \quad[-0.02,0.07]$

Health Stress $\quad 0.27 \quad[0.23,0.31] \quad 0.26 \quad[0.22,0.30]$

Financial Stress $\quad 0.02 \quad[-0.02,0.05] \quad-0.03 \quad[-0.07,0.01]$ 